\title{
10 most important contemporary challenges in the management of SLE
}

\author{
Renaud Felten, ${ }^{1,2}$ Flora Sagez, ${ }^{1,2}$ Pierre-Edouard Gavand, ${ }^{2,3}$ Thierry Martin, ${ }^{2,3}$ \\ Anne-Sophie Korganow, ${ }^{2,3}$ Christelle Sordet, ${ }^{1,2}$ Rose-Marie Javier, ${ }^{1,2}$ \\ Pauline Soulas-Sprauel, ${ }^{2,3}$ Marianne Rivière, ${ }^{4}$ Florence Scher, ${ }^{5}$ Vincent Poindron, ${ }^{2,3}$ \\ Aurélien Guffroy, ${ }^{2,3}$ Laurent Arnaud ${ }^{1,2}$
}

To cite: Felten $\mathrm{R}$, Sagez F, Gavand P-E, et al. 10 most important contemporary challenges in the management of SLE. Lupus Science \& Medicine 2019;6:e000303. doi:10.1136/ lupus-2018-000303

RF and FS contributed equally.

Received 17 October 2018 Revised 12 December 2018 Accepted 16 December 2018

Check for updates

(c) Author(s) (or their employer(s)) 2019. Re-use permitted under CC BY-NC. No commercial re-use. See rights and permissions. Published by BMJ.

${ }^{1}$ Service de rhumatologie, Hôpitaux Universitaires de Strasbourg, Université de Strasbourg, Strasbourg, France ${ }^{2}$ Centre National de Références des Maladies Systémiques et Autoimmunes Rares Est Sud-Ouest (RESO)-LUPUS, Strasbourg, France

${ }^{3}$ Service d'immunologie clinique, Hôpitaux Universitaires de Strasbourg, Université de

Strasbourg, Strasbourg, France

${ }^{4}$ Association Française du Lupus et autres Maladies AutoImmunes (AFL+), France

${ }^{5}$ Service de Pharmacie-

Stérilisation, Hôpitaux

Universitaires de Strasbourg,

Strasbourg, France

Correspondence to

Pr Laurent Arnaud; laurent. arnaud@chru-strasbourg.fr

\section{ABSTRACT}

From a 1-year survival of less than $50 \%$ before the discovery of glucocorticoids to over $90 \%$ at 10 years in most dedicated centres, the spectrum of SLE has profoundly evolved. Despite this improvement, several major challenges currently remain. The aim of this review is to analyse what are, according to us, the 10 most important contemporary challenges in the management of SLE. Among those are the need to treat to target to favour disease remission (or low disease activity), limit the use of glucocorticoids, derive more comprehensive tools for the evaluation of disease activity, develop more effective drugs (yielding successful trials), dissect the heterogeneity of the disease both at the molecular and genetic levels, identify relevant biomarkers for individualised treatment, manage fertility and pregnancy, tackle comorbidities such as cardiovascular risk, the prevention of infections and osteoporosis, improve the network of care (from the patients' perspective), and favour a holistic approach (integrating fatigue, adherence to treatment, physical activity). Altogether, these 10 contemporary challenges in SLE may be considered as a roadmap for those involved in the daily care of patients with SLE, as well as for researchers who may wish to contribute to an improved management of this rare and complex disease.

\section{INTRODUCTION}

Despite the improvement of pharmacopoeia and disease prognosis, ${ }^{1}$ numerous challenges remain for the management of SLE (box 1). The aim of this review was to analyse what are, according to us, the 10 most important contemporary challenges in the management of SLE. For this, the multidisciplinary team of clinicians, pharmacists and researchers from our national reference centre for rare autoimmune and systemic diseases (Strasbourg, France), as well as the French SLE patient association, selected the 10 most important contemporary challenges in management of SLE. A round table was conducted so that each coauthor would give his expertise on those challenges. All those proposals were extensively discussed, refined and eventually grouped together by theme. Our ability to further improve the prognosis of the disease as well as patients' quality of life will definitely result from our ability to tackle these challenges.

\section{Favouring disease remission (or low disease activity)}

Among the biggest challenges for the clinician are the early detection of flares as well as the need to confirm that apparently inactive patients are truly in remission. This led to the introduction of the treat-to-target strategy for SLE which identified remission as one of the most important targets in SLE, while it was recognised at the same time that there was no clear definition for it. ${ }^{2}$ In 2016 an initiative was undertaken by a large international multiparty panel to achieve a consensus on the definition of remission in SLE (Definitions of Remission in SLE (DORIS)). ${ }^{3}$ This led to an agreement on three principles: remission should be a durable state (however, no consensus was reached on the minimum duration); a validated index should be used; and a distinction should be made between remission off and on therapy. At the same time and for similar reasons, the Asia Pacific Lupus Collaboration group developed a Low Disease Activity State index. We believe that one of the main challenges is to validate whether these definitions are indeed predictive of outcomes, including damage, death, recurrent flares and health-related quality of life measures, and whether they can be used as clinical trial outcomes. Preliminary results seem to indicate that they do at least for damage accrual.

\section{Limiting the use of glucocorticoids}

Glucocorticoids (GCs) remain a cornerstone in the treatment of SLE, especially in the case of severe manifestations, and have played a major role in the overall improvement of SLE prognosis. In a recent survey by the Systemic Lupus International Collaborating Clinics (SLICC) group, almost 33\% of 
Box 1 The 10 main contemporary challenges in SLE

T2T: favouring disease remission (or low disease activity)

- Limiting the use of glucocorticoids.

- Deriving more comprehensive tools for the evaluation of disease activity.

- Developing more effective (and better tolerated) drugs.

Dissecting the heterogeneity of the disease at the molecular and genetic levels.

- Identifying relevant biomarkers for individualised treatment.

- Managing fertility and pregnancy.

- Managing comorbidities.

- Improving the network of care (the patients' perspective).

- Favouring a holistic approach.

T2T, treat to target.

patients never discontinued GCs after a mean follow-up of 7.26 years. ${ }^{4}$ Several studies have emphasised the risk of damage accrual in a patient with SLE treated with GC, including at low doses such as $5 \mathrm{mg} /$ day or $7.5 \mathrm{mg}$ / day of prednisone-equivalent. ${ }^{5}$ The initial GC dose is a strong predictor of the overall GC exposure independent of initial disease activity, with potential overtreatment in those with limited disease activity. ${ }^{6}$ From these findings rise several challenges about the current and future use of GCs in SLE. First, the use of a low dose of GC (such as $5 \mathrm{mg}$ /day) or a GC-free maintenance regimen should be discussed as a major target. ${ }^{3}$ Second, GC management should be an important concern in future randomised controlled trial: GC tapering scheme, GC-related adverse events, damage accrual and cumulative GC doses should be included in the assessment. Third, medical practice ${ }^{7}$ should be implemented in such a way that GC doses are managed using more objective tools (such as the GC cumulative dose, follow-up of disease activity and damage, and recording of GC-related adverse events).

\section{Deriving more comprehensive tools for the evaluation of disease activity}

Behind the simple concept of disease activity in SLE hides a complex multidimensional reality, where the specific clinical manifestations attributed to SLE by the physician, the subjective experiences of the patient and the efficacy of treatments interact altogether. ${ }^{3}$ For some organ manifestations, well-established disease-specific measures exist to assess disease activity in that organ (eg, the Cutaneous Lupus Erythematosus Disease Area and Severity Index ${ }^{8}$ ); for others, assessment of disease activity relies on common clinical skills or analyses as those used in routine clinical practice. Besides assessing disease activity of a specific organ system, several tools have been developed to assess the overall activity of the disease. Those include the Systemic Lupus Erythematosus Disease Activity Index $\left(\mathrm{SLEDAI}^{9}\right)$ and its evolutions, the British Isles Lupus Assessment Group (BILAG) and its revision, ${ }^{10}$ the European Consensus Lupus Assessment Measure, the Lupus Activity Index, the Systemic Lupus Activity Measure and its revision, and the Physician's Global Assessment (PGA) by visual analogue scale. More recently, novel composite outcomes such as the SLE Responder Index, which is based on the improvement of the SLEDAI with no worsening in BILAG and PGA, have appeared. ${ }^{11}$ In most cases the clinician has to form a judgement with regard to whether each manifestation is due to SLE or not, and in actual practice this can be a major challenge. We believe that we should search for more objectivity and reproducibility in our way to assess disease activity, such as by discovering new biomarkers that would better reflect disease status, and promote the use of large-scale, data-driven approaches, such as deep machine learning, to identify as early as possible unfavourable individual patient trajectories among large cohorts of patients.

\section{Developing more effective drugs}

There have been many advances in the therapeutic management of SLE. ${ }^{12} 13$ With those has occurred a profound change in the prognosis of the disease. ${ }^{1}$ However, this does not hinder the fact that several gaps remain in the care of patients with SLE. For example, in lupus nephritis, a significant proportion of patients progress towards end-stage kidney disease. ${ }^{14}$ In the Aspreva Lupus Management Study ${ }^{15}$ the primary efficacy endpoint was only achieved in $56.2 \%$ of patients receiving mycophenolate mofetil. This illustrates the need for more efficacious drugs and new tools to evaluate response to therapy. In neuropsychiatric SLE, many efforts have been made to guide best practice for the diagnosis and management, but there is only weak evidence on which to base recommendations. ${ }^{16}$ Our group has recently published a systematic review of 74 targeted therapies for $\mathrm{SLE},{ }^{2}$ showing that we may expect great changes in the therapeutic tools available for SLE treatment. We believe that current challenges are shifting from whether some new drugs will be available to the identification of the best strategy for the selection of the most adequate drug (or drug combination) at the patient level, to warrant a positive balance between efficacy and side effects. The need to investigate biomarkers that would allow adequate prediction of response to therapy remains high, but when solved will allow a more rational selection of the optimal pharmacological agent within the broad pipeline of targeted therapies for SLE.

\section{Dissecting the heterogeneity of the disease at the molecular and genetics levels}

Environmental factors play a significant role in SLE development and exacerbation. ${ }^{17}$ In addition, a major genetic contribution to susceptibility to SLE is clearly supported by an increased concordance rate in monozygotic twins $(>35 \%) .{ }^{18}$ Importantly, the genetic component to the pathogenesis of SLE is very complex because most patients have polygenic disease. ${ }^{18-20}$ Therefore, this complex genetic contribution to SLE, in synergy with environmental factors, reflects the implication of different biological pathways that participate in the 
pathophysiology of the disease and its high heterogeneity. ${ }^{170} 21$ Since 2008, genome-wide association studies (GWAS) have described almost 100 susceptibility single nucleotide polymorphisms for SLE. ${ }^{19}{ }^{20}$ Almost all variants have ORs of less than 1.5, making them of unproven clinical utility in the diagnosis or in the treatment of patients. In addition, the biological impact is known for only 20 of those variants. Despite this, GWAS have helped to draw a complex model for SLE pathophysiology. Importantly, the patients included in GWAS present different clinical manifestations but are grouped within the same 'bracket'. This leads to an oversimplification of the disease status. Also, variants identified by GWAS explain only a fraction of the overall heritability of SLE. Therefore, there is a missing heritability which could be explained notably by epigenetics and by expression quantitative trait loci. ${ }^{19} 20$ Finally, although very rare, the monogenic forms must be considered in the study of SLE genetics because we could imagine that less deleterious variants in the genes implicated in monogenic forms could explain the pathophysiology of SLE in polygenic patients. In conclusion, behind the important heterogeneity of SLE clinical manifestations reside a high number of molecular mechanisms, linked to a complex genetic heritability and expression. One important challenge is to develop an optimal model for patients' substratification using multiomics to allow the development of personalised medicine for patients with SLE.

\section{Identifying relevant biomarkers for individualised treatment} Finding the right treatment for the right patient remains one of the most important challenges in SLE. The markers that have been identified for decades, such as antidouble-stranded-DNA IgG antibodies, complement factor consumption or leucopaenia, are now insufficient to progress in the management of the disease. The era of multiomics (genomics, transcriptomics, proteomics or metabolomics), by means of high-throughput tools such as next-generation sequencing and computerisation of data, opens the door for integrated and individualised approach. ${ }^{22}$ At a proteomic level, the imbalance of cytokines could be used as potential biomarkers. The most emblematic example is type I interferon signature found in the sera of almost $70 \%$ of patients with SLE. ${ }^{23}$ However, the precise contribution of each interferon-stimulated gene to the phenotype is not yet fully understood. In a longitudinal study of 158 paediatric patients with SLE, ${ }^{24}$ the plasmablast signature appears to be one of the best biomarkers of disease activity, while the neutrophil signature correlated well with nephritis. This kind of study opens the door for personalised regimens. Proinflammatory molecules such as interleukin (IL)-6, IL-17, but also cytokines such as B-cell activating factor (BAFF) or IL-2, also represent a major topic of research ${ }^{25}$ Each of these cytokine pathways can be targeted by biologics or recombinant molecules. Finally, possible emerging biomarkers could arise from the world of non-coding RNAs, which are tissue-specific regulators of gene expression that have been proposed as markers of flares. One of the main current challenges is to develop a holistic approach of the disease and therefore to integrate the vast amount of available data at the patient level. This will undoubtedly require some specialised structures to interface all the players involved in this new era of care: clinical specialists, advanced diagnosis physicians, researchers in biomedicine and data scientists.

\section{Managing fertility and pregnancy}

Pregnancy has always been challenging for patients with SLE and their treating physicians. In general, a multidisciplinary team, consisting of a rheumatologist or an internist and an obstetrician with significant experience on high-risk pregnancies, manages the care of pregnant patients with SLE. Patients should be informed that pregnancies in SLE should be carefully anticipated, and that prepregnancy multidisciplinary counselling is important to determine the risk of both maternal and fetal complications. ${ }^{26}$ SLE is usually not associated with infertility unless the patient has been treated with cyclophosphamide. Ovarian protection using Gonadotropin Releasing Hormone (GnRH) agonists can be administered during cytotoxic treatment but induces an artificial menopause which is not always well accepted by young patients. Ovarian preservation (cryopreservation of ovarian tissue or of mature oocytes) can sometimes be difficult to implement in the context of a relative emergency or in case of thrombocytopaenia and/or anticoagulation. The overall prognosis of pregnancy in SLE is more favourable when the disease has been quiescent for at least 6 months, even 1 year in nephritis, with a low organ damage score. Active SLE at the time of conception and/or positivity for lupus anticoagulant or triple positivity, use of antihypertensive treatments, and low platelet count are strong predictors of complicated maternal and obstetrical outcomes. The management of SLE during pregnancy is challenging because pregnancy complications can mimic SLE flares ${ }^{27}$; antiphospholipid antibodies (aPLs) can lead to both maternal and fetal adverse events, while anti-Ro/SSA or anti-La/SSB antibodies are associated with congenital heart block or neonatal lupus; we should take into account the potential teratogenic effects of some of SLE treatments. Among the main challenges, we have to improve the outcomes of pregnancy in patients with aPL and/or anti-SSA/B antibodies.

\section{Managing comorbidities \\ The cardiovascular risk}

Early mortality in SLE remains mostly related to disease activity, but the frequency of late cardiovascular morbimortality is increasing in line with prolonged overall patient survival. ${ }^{28}$ Multiple risk factors contribute to this, such as the classical cardiovascular risk factors (CVRF), the disease itself through its activity, treatments and complications, and the thrombotic risk due to aPLs. Observational studies suggest that most classical CVRFs are insufficient to explain the increased cardiovascular 
risk by themselves, leading to the concept of 'accelerated atherosclerosis' ${ }^{29}{ }^{30}$ Given this high risk, a group of French experts made recommendations for the management of cardiovascular risk factors in patients with SLE. ${ }^{28}$ One of the challenges we have to face is that studies are in many cases based on the North American population and it is not clear whether those data can be extrapolated to European populations. We believe that another challenge is that validated tools for assessing cardiovascular risk in the general population are not adapted to the estimation of the cardiovascular risk in SLE. This generally leads to an underestimation of the actual risk. It is therefore crucial to develop adequate scores. Finally, we should conduct new therapeutic trials to provide more evidence-based data on how to manage cardiovascular risk in patients with SLE.

\section{The prevention of infection}

Infections remain one of the major causes of morbidity and mortality in SLE. ${ }^{31}$ Infections are statistically associated with the use of GCs and immunosuppressive therapy, lymphopaenia and hypocomplementaemia, but severe organ involvement such as lupus nephritis is also a major risk factor. Recommendations for vaccination in adult patients with autoimmune inflammatory rheumatic diseases have been published in 2011 by the European League Against Rheumatism ${ }^{32}$ and more recently in patients with SLE by the French health authorities. Vaccine strategy against pneumococcus is based on the 13-valent pneumococcal protein-conjugate vaccine followed by the 23-valent pneumococcal polysaccharide vaccine (PPS23) at least 8 weeks later. However, a randomised controlled trial (using a 7-valent vaccine) showed that sequential administration was not superior to the PPS23 vaccine alone in terms of immunogenicity. ${ }^{33}$ Live attenuated vaccines are contraindicated in patients with GCs at daily dosage of more than $10 \mathrm{mg}$ of prednisone, immunosuppressive therapy and/or biotherapy. Inactivated vaccines can be used at any time; however, their immunogenicity may be substantially reduced overall in active SLE disease and/or under immunosuppressive treatment and/or in case of lymphopaenia. The theoretical risk of a flare of SLE after vaccination has never been formally confirmed. Vaccination rates remain low in SLE, in particular for vaccine against pneumococcus and influenza. ${ }^{34}$ Significant efforts are urgently needed to reinforce the immunisation coverage. Vaccination when patients are not yet or no longer treated with high-dose corticosteroids and/ or immunosuppressive drugs would improve the immunogenicity of the vaccine. We believe that new vaccine strategies need to be evaluated and validated specifically in SLE.

\section{The management of osteoporosis}

Osteoporosis is a source of chronic pain and disability which contributes to morbidity and is a major challenge to the quality of life. SLE is significantly associated with lower bone mineral density (BMD) levels and with an increased fracture risk at all sites, especially vertebral fractures, ${ }^{35-37}$ which are often asymptomatic. ${ }^{35}$ The aetiology of bone loss and fractures is multifactorial in SLE, including GC therapy, disease activity, early menopause (such as in patients treated with intravenous cyclophosphamide), vitamin D deficiency (related to photoprotection) and frailty. ${ }^{35}$ We believe that a thorough assessment of bone status is essential at the time of SLE diagnosis. This should include an evaluation of dietary calcium intake, consumption of bone toxics (tobacco, alcohol and excess salt intake), a search for risk factors for fracture risk estimation (personal history of low trauma fracture) as well as a systematic measurement of BMD in all patients with SLE, including men and children aged $7+{ }^{36}$ If BMD is abnormal, an X-ray examination of the dorsal and lumbar spine should be performed, searching for asymptomatic prevalent fractures. ${ }^{35}$ The detection of one or more asymptomatic prevalent vertebral fracture with at least osteopaenia is an indication for antiosteoporotic treatment. A true challenge is that minimisation of the dose and the duration of GC must be a priority associated with adequate calcium and vitamin $\mathrm{D}$ intake (25-hydroxy vitamin $\mathrm{D}$ above $30 \mathrm{ng} / \mathrm{mL}$ ).

\section{Improving the network of care (the patients' perspective, by the Association Française Du lupus et autres maladies autoimmunes, AFL+)}

Rare diseases, including SLE, have been recognised as one of the five top health priorities for France in the 2004 French law of public health. In 2005, the launch of the first 'Plan national maladies rares' (national plan for rare diseases) by the French Ministry of Health has strongly boosted the recognition of SLE as a rare disease and led to a new organisation of the French health system, with the creation of the national reference centre for rare autoimmune and systemic diseases. While this initiative has greatly improved the network of care for patients with SLE, several challenges remain, such as improving more early diagnosis and limiting diagnosis uncertainty. In that sense, the development of multidisciplinary expertise, including that of telemedicine, is crucial to promote patient access to care and adapted treatment. The relationship between hospitals and general practitioners should be developed and the training of health professional for the recognition of rare diseases, including SLE, should be encouraged. The frequent release of updated national guidelines, such as the PNDS ('Protocole National de Diagnostic et de Soins', National Diagnostic and Care Protocols), should be encouraged, as this may help in limiting diagnosis error and improving the overall care of patients with SLE according to validated national referentials. A dedicated diagnostic consultation should be incorporated into the patient journey within the health system, with a role for psychological counselling and patient association. Therapeutic education for the patients, their family and those who support them should be promoted. Altogether, the interaction between the patients, their physicians and the patient organisations 
should be developed at both national and international levels. Overall, a global awareness about lupus can be further improved by organising dedicated events such as the European Lupus Day (Strasbourg, 10 May 2019).

\section{Favouring a holistic approach}

Many aspects of SLE, such as fatigue, treatment adherence or smoking cessation, should be taken care of simultaneously, using a holistic approach. Patient-reported outcome (PRO) measures have been designed to capture patient perceptions of their health condition, health-related quality of life, well-being and other aspects. PROs encompass domains such as pain, physical function, fatigue, anxiety and depression, among many others. The use of PRO in daily clinical practice is far to be generalised and may help to better characterise the impact of the disease on patients' life. Fatigue is a frequent complaint of patients with SLE (up to $90 \%$ of patients) and is more prevalent in SLE compared with healthy controls. ${ }^{38}$ It has an important impact on the quality of life of patients with SLE and is a major cause of work disability. ${ }^{39}$ Fatigue is a complex multidimensional symptom and its management is challenging. The role of disease activity remains controversial, while the impact of depression and anxiety is strong, and that of fibromyalgia, pain, obesity and sleep disorders significant. ${ }^{40}$ Organic causes of fatigue such as thyroid disease or adrenal insufficiency should also be considered. Only a few trials were designed specifically to treat fatigue in SLE. However, fatigue is often assessed as a secondary endpoint in phase II or III trials. We believe that we should conduct more and better designed trials to evaluate non-pharmacological interventions (such as physical activity and psychosocial support) as well as pharmacological interventions for the reduction of fatigue in SLE, targeting disease activity and other dimensions such as depression and anxiety. In SLE, the percentage of non-adherent patients can be as high as $68 \%$. The main predictors of non-adherence are a non-white ethnic background, low socioeconomic level, lower education level, polymedication, mood disorders such as depression and rural residency. ${ }^{41}$ It is however neither demonstrated that the adherence is related to patients' knowledge of the disease nor to the trust in the doctor. Non-adherence contributes to worse patient outcomes, with significantly higher number of disease flares, ${ }^{41}{ }^{42}$ visits in emergency departments and outpatient rheumatology visits. Hydroxychloroquine withdrawal is associated with an increased risk of flare. ${ }^{43}$ A very low blood concentration of hydroxychloroquine $(<200 \mathrm{ng} / \mathrm{mL})$ is a good marker of poor adherence and may be useful to discriminate between failure of hydroxychloroquine and non-adherent patients. ${ }^{44}$ It is crucial to talk with the patients about their difficulties related to treatments. We strongly believe that providing broader access to educational material about treatment and to education programmes should be encouraged to increase adherence. Disease activity and severity is markedly increased in SLE smokers, compared with non-smokers. ${ }^{45}$ It is therefore crucial to encourage patients with SLE to stop use of tobacco. The use of dedicated communication techniques such as motivational interviewing can be useful, by bringing patients to self-identify the most beneficial behaviours for their health ('inherent motivation'). We believe that we encourage the use of motivational techniques to limit the impact of smoking. The health benefits of physical activity are well established. These include lower cardiovascular risk and positive effects on mental health. In SLE, physical inactivity is common, with up to $72 \%$ of patients not meeting the WHO recommendations. ${ }^{46}$ The main factors associated to sedentary time are age, disease activity, musculoskeletal manifestations, depression, fatigue and fibromyalgia. Systematic reviews suggested that exercise reduces fatigue and depression, and improves cardiorespiratory capacity without affecting disease activity. ${ }^{478} \mathrm{We}$ believe it is important to encourage regular practice of physical activities in SLE.

\section{CONCLUSION}

Altogether, these 10 contemporary challenges may be considered as a roadmap for those involved in the daily care of patients with SLE, as well as those researchers and health policymakers who wish to contribute to an improved management of this rare and complex disease.

Contributors LA: article design, writing of section 3 (deriving more comprehensive tools for the evaluation of disease activity), manuscript drafting and revision. RF: writing of section 4 (developing more effective [and better tolerated]) drugs, manuscript revision. FSa: writing of section 8 (managing comorbidities). P-EG: writing of section 10 (favouring a holistic approach). TM: writing of section 1 (T2T: favouring disease remission [or low disease activity]), manuscript revision. A-SK: writing of section 7 (managing fertility and pregnancy), manuscript revision. CS: writing of section 10 (favouring a holistic approach). R-MJ: writing of section 8 (managing comorbidities). PS-S: writing of section 5 (dissecting the heterogeneity of the disease at the molecular and genetic levels). MR: writing of section 9 (improving the network of care [the patients' perspective]). FSc: writing of section 4 (developing more effective [and better tolerated] drugs). VP: writing of section 2 (limiting the use of glucocorticoids). AG: writing of section 6 (identifying relevant biomarkers for individualised treatment).

Funding The authors have not declared a specific grant for this research from any funding agency in the public, commercial or not-for-profit sectors.

Competing interests LA has received honoraria and/or funding from Amgen, AstraZeneca, GSK, Janssen-Cilag, LFB, Lilly, Menarini France, Novartis, Pfizer, Roche-Chugaï and UCB. RF has received honoraria from AbbVie, BMS and Pfizer. MR has received funding from GSK and Boehringer Ingelheim. TM has received honoraria and/or funding from Amgen, GSK, CSL Behring, Janssen-Cilag, Lilly, Novartis, Pfizer, Roche-Chugaï and Sanofi-Genzyme.

Patient consent for publication Not required.

Provenance and peer review Not commissioned; externally peer reviewed. Data sharing statement Not available.

Open access This is an open access article distributed in accordance with the Creative Commons Attribution Non Commercial (CC BY-NC 4.0) license, which permits others to distribute, remix, adapt, build upon this work non-commercially, and license their derivative works on different terms, provided the original work is properly cited, appropriate credit is given, any changes made indicated, and the use is non-commercial. See: http://creativecommons.org/licenses/by-nc/4.0/.

\section{REFERENCES}

1. Arnaud L, Fagot J-P, Mathian A, et al. Prevalence and incidence of systemic lupus erythematosus in France: a 2010 nation-wide population-based study. Autoimmun Rev 2014;13:1082-9. 
2. van Vollenhoven RF, Mosca M, Bertsias G, et al. Treat-to-target in systemic lupus erythematosus: recommendations from an international Task Force. Ann Rheum Dis 2014;73:958-67.

3. van Vollenhoven R, Voskuyl A, Bertsias G, et al. A framework for remission in SLE: consensus findings from a large international Task Force on definitions of remission in SLE (DORIS). Ann Rheum Dis 2017;76:554-61.

4. Little J, Parker B, Lunt M, et al. Glucocorticoid use and factors associated with variability in this use in the systemic lupus international collaborating clinics inception cohort. Rheumatology 2018;57:677-87.

5. Apostolopoulos D, Kandane-Rathnayake R, Raghunath S, et al. Independent association of glucocorticoids with damage accrual in SLE. Lupus Sci Med 2016;3:e000157.

6. Ruiz-Irastorza G, Garcia M, Espinosa G, et al. First month prednisone dose predicts prednisone burden during the following 11 months: an observational study from the RELES cohort. Lupus Sci Med 2016;3:e000153.

7. Emamikia S, Gentline C, Chatzidionysiou K, et al. Relationship between glucocorticoid dose and adverse events in systemic lupus erythematosus: data from a randomized clinical trial. Scand J Rheumatol 2018;47:131-40.

8. Albrecht J, Taylor L, Berlin JA, et al. The CLASI (cutaneous lupus erythematosus disease area and severity Index): an outcome instrument for cutaneous lupus erythematosus. J Invest Dermatol 2005;125:889-94.

9. Bombardier C, Gladman DD, Urowitz MB, et al. Derivation of the SLEDAI. A disease activity index for lupus patients. the Committee on prognosis studies in SLE. Arthritis Rheum 1992;35:630-40.

10. Isenberg DA, Rahman A, Allen E, et al. BILAG 2004. Development and initial validation of an updated version of the British Isles lupus Assessment Group's disease activity index for patients with systemic lupus erythematosus. Rheumatology 2005;44:902-6.

11. Navarra SV, Guzmán RM, Gallacher AE, et al. Efficacy and safety of belimumab in patients with active systemic lupus erythematosus: a randomised, placebo-controlled, phase 3 trial. Lancet 2011;377:721-31.

12. Chasset $F$, Arnaud $L$. Targeting interferons and their pathways in systemic lupus erythematosus. Autoimmun Rev 2018;17:44-52.

13. Felten R, Dervovic E, Chasset $F$, et al. The 2018 pipeline of targeted therapies under clinical development for systemic lupus erythematosus: a systematic review of trials. Autoimmun Rev 2018:17:781-90.

14. Tunnicliffe DJ, Palmer SC, Henderson L, et al. Immunosuppressive treatment for proliferative lupus nephritis. Cochrane Database Syst Rev 2018;6:CD002922.

15. Appel GB, Contreras G, Dooley MA, et al. Mycophenolate mofetil versus cyclophosphamide for induction treatment of lupus nephritis. J Am Soc Nephrol 2009;20:1103-12.

16. Fernandes Moça Trevisani V, Castro AA, Ferreira Neves Neto J, et al. Cyclophosphamide versus methylprednisolone for treating neuropsychiatric involvement in systemic lupus erythematosus. Cochrane Database Syst Rev 2013;2:CD002265.

17. Tsokos GC, Lo MS, Costa Reis P, et al. New insights into the immunopathogenesis of systemic lupus erythematosus. Nat Rev Rheumatol 2016;12:716-30.

18. Moser KL, Kelly JA, Lessard CJ, et al. Recent insights into the genetic basis of systemic lupus erythematosus. Genes Immun 2009;10:373-9.

19. Teruel M, Alarcón-Riquelme ME. The genetic basis of systemic lupus erythematosus: what are the risk factors and what have we learned. $J$ Autoimmun 2016;74:161-75.

20. Deng Y, Tsao BP. Updates in lupus genetics. Curr Rheumatol Rep 2017;19:68.

21. Zharkova O, Celhar T, Cravens PD, et al. Pathways leading to an immunological disease: systemic lupus erythematosus. Rheumatology 2017;56(suppl_1):i55-i66.

22. Moulton VR, Suarez-Fueyo A, Meidan E, et al. Pathogenesis of human systemic lupus erythematosus: a cellular perspective. Trends in Molecular Medicine 2017;23:615-35.

23. Banchereau J, Pascual V. Type I interferon in systemic lupus erythematosus and other autoimmune diseases. Immunity 2006;25:383-92.

24. Banchereau R, Hong S, Cantarel B, et al. Personalized Immunomonitoring uncovers molecular networks that stratify lupus patients. Cell 2016;165:551-65.

25. Steri M, Orrù V, Idda ML, et al. Overexpression of the cytokine BAFF and autoimmunity risk. N Engl J Med 2017;376:1615-26.
26. Buyon JP, Kim MY, Guerra MM, et al. Predictors of pregnancy outcomes in patients with lupus. Ann Intern Med 2015;163:153-63.

27. Kim MY, Guerra MM, Kaplowitz E, et al. Complement activation predicts adverse pregnancy outcome in patients with systemic lupus erythematosus and/or antiphospholipid antibodies. Ann Rheum Dis 2018;77:549-55.

28. Arnaud L, Mathian A, Adoue D, et al. [Screening and management of cardiovascular risk factors in systemic lupus erythematosus: Recommendations for clinical practice based on the literature and expert opinion]. Rev Med Interne 2015;36:372-80.

29. Esdaile JM, Abrahamowicz M, Grodzicky T, et al. Traditiona Framingham risk factors fail to fully account for accelerated atherosclerosis in systemic lupus erythematosus. Arthritis Rheum 2001;44:2331-7.

30. Urowitz MB, Ibanez D, Gladman DD. Atherosclerotic vascular events in a single large lupus cohort: prevalence and risk factors. $J$ Rheumatol 2007;34:70-5.

31. Feldman CH, Hiraki LT, Winkelmayer WC, et al. Serious infections among adult Medicaid beneficiaries with systemic lupus erythematosus and lupus nephritis. Arthritis Rheumatol 2015:67:1577-85.

32. van Assen S, Agmon-Levin N, Elkayam O, et al. EULAR recommendations for vaccination in adult patients with autoimmune inflammatory rheumatic diseases. Ann Rheum Dis 2011;70:414-22.

33. Grabar S, Groh M, Bahuaud M, et al. Pneumococcal vaccination in patients with systemic lupus erythematosus: a multicenter placebo-controlled randomized double-blind study. Vaccine 2017:35:4877-85.

34. Lawson EF, Trupin L, Yelin EH, et al. Reasons for failure to receive pneumococcal and influenza vaccinations among immunosuppressed patients with systemic lupus erythematosus. Semin Arthritis Rheum 2015;44:666-71.

35. Bultink IEM. Bone Disease in Connective Tissue Disease/Systemic Lupus Erythematosus. Calcif Tissue Int 2018;102:575-91.

36. Lim SH, Benseler SM, Tyrrell PN, et al. Low bone mineral density is present in newly diagnosed paediatric systemic lupus erythematosus patients. Ann Rheum Dis 2011;70:1991-4.

37. Wang X, Yan S, Liu C, et al. Fracture risk and bone mineral density levels in patients with systemic lupus erythematosus: a systematic review and meta-analysis. Osteoporos Int 2016;27:1413-23.

38. Cleanthous S, Tyagi M, Isenberg DA, et al. What do we know about self-reported fatigue in systemic lupus erythematosus? Lupus 2012;21:465-76.

39. Ahn GE, Ramsey-Goldman R. Fatigue in systemic lupus erythematosus. Int J Clin Rheumtol 2012;7:217-27.

40. Burgos PI, Alarcón GS, McGwin G, et al. Disease activity and damage are not associated with increased levels of fatigue in systemic lupus erythematosus patients from a multiethnic cohort: LXVII. Arthritis Rheum 2009;61:1179-86.

41. Feldman $\mathrm{CH}$, Collins J, Zhang Z, et al. Dynamic patterns and predictors of hydroxychloroquine nonadherence among Medicaid beneficiaries with systemic lupus erythematosus. Semin Arthritis Rheum 2018;48:205-13.

42. Mehat P, Atiquzzaman M, Esdaile JM, et al. Medication nonadherence in systemic lupus erythematosus: a systematic review. Arthritis Care Res 2017;69:1706-13.

43. Tsakonas E, Joseph L, Esdaile JM, et al. A long-term study of hydroxychloroquine withdrawal on exacerbations in systemic lupus erythematosus. The Canadian hydroxychloroquine Study Group. Lupus 1998;7:80-5.

44. Costedoat-Chalumeau N, Amoura Z, Hulot J-S, et al. Very low blood hydroxychloroquine concentration as an objective marker of poor adherence to treatment of systemic lupus erythematosus. Ann Rheum Dis 2007;66:821-4.

45. Chasset $F$, Francès $C$, Barete $S$, et al. Influence of smoking on the efficacy of antimalarials in cutaneous lupus: a meta-analysis of the literature. J Am Acad Dermatol 2015;72:634-9.

46. Mancuso CA, Perna M, Sargent AB, et al. Perceptions and measurements of physical activity in patients with systemic lupus erythematosus. Lupus 2011;20:231-42.

47. Margiotta DPE, Basta F, Dolcini G, et al. Physical activity and sedentary behavior in patients with systemic lupus erythematosus. Plos One 2018;13:e0193728.

48. O'Dwyer T, Durcan L, Wilson F. Exercise and physical activity in systemic lupus erythematosus: a systematic review with metaanalyses. Semin Arthritis Rheum 2017:47:204-15. 